\title{
Plasticidad muscular y entrenamiento concurrente: implicaciones para la prescripción del ejercicio
}

\author{
Muscle Plasticity and Concurrent Training: \\ implications to exercise prescription
}

* Guilherme Rosa

Rosa, G. (2019). Plasticidad muscular y entrenamiento concurrente: implicaciones para la prescripción del ejercicio. Revista Ciencias de la Actividad Física UCM, N²0(2), julio-diciembre, 1-9. DOI: http://doi. org/10.29035/rcaf.20.2.7

\section{RESUMEN}

Con el propósito de discutir los efectos del entrenamiento concurrente sobre las características del músculo esquelético mediadas por la plasticidad muscular, así como los aspectos relacionados con la prescripción de este tipo de ejercicio, se realizó una revisión de la literatura sobre el tema. Se utilizaron catorce referencias obtenidas de las bases de datos PubMed, SciELO y Schoolar Google a través de las siguientes palabras clave: "entrenamiento concurrente, ejercicio concurrente, plasticidad muscular, ejercicio físico". La plasticidad muscular puede reducir las adaptaciones inducidas por las modalidades que conforman el entrenamiento concurrente. Por lo tanto, al planificar el programa de entrenamiento, es necesario observar estrategias que busquen minimizar este efecto.

\section{Palabras clave}

Entrenamiento concurrente, plasticidad muscular, ejercicio físico.

* Grupo de Pesquisas em Exercício Físico e Promoção da Saúde - UCB/RJ; Faculdade Bezerra de Araújo - FABA/RJ. Rio de Janeiro, Brasil. 


\section{ABSTRACT}

In order to discuss the effects of concurrent training on skeletal muscle characteristics mediated by muscle plasticity, as well as aspects related to the prescription of this type of exercise, a review of the literature on the subject was made. Fourteen references obtained from the PubMed, SciELO and Schoolar Google databases following the keywords "concurrent training, concurrent exercise, muscular plasticity, physical exercise" were used. Muscle plasticity can reduce the adaptations induced by the modalities that make up concurrent training. Therefore, when planning the training program, it is necessary to observe strategies that seek to minimize this effect.

\section{Key words}

Concurrent training, muscle plasticity, physical exercise.

\section{Introducción}

El músculo esquelético es un tejido dinámico con la habilidad intrínseca de adaptarse a los estímulos ambientales como resultado de cambios cualitativos y cuantitativos en la expresión génica, siendo esta capacidad definida como plasticidad (Mounier, Théret, Lantier, Foretz, \& Viollet, 2015). Entre los factores que intervienen en las características del tejido muscular, está el estímulo recibido a través del ejercicio físico (Hoppeler, 2016; Powers \& Howley, 2014). El entrenamiento concurrente combina el entrenamiento cardiorrespiratorio y neuromuscular en un mismo programa de entrenamiento, generando la posibilidad de que cada modalidad estimule adaptaciones específicas (de Sena Martins et al., 2017). Debido a sus particularidades, esta forma de entrenamiento ha sido objeto de investigaciones con diferentes objetivos y poblaciones (Monteiro-Lago et al., 2019; Della Corte et al., 2019; Farías, Borba-Pinheiro, Oliveira \& Vale, 2014; Rosa, Dantas, Biehl, Castro e Silva, Montano \& Mello, 2012).

En este artículo, en formato de comentario, se abordarán las principales vías de señalización intracelulares en el músculo esquelético, la influencia de las características de cada una de las modalidades que componen el entrenamiento concurrente sobre tales vías, así como las principales consideraciones a ser observadas para la prescripción del ejercicio físico con el fin de minimizar la interferencia negativa que una vía de señalización puede ejercer sobre la otra. 
Los artículos utilizados en el trabajo fueron seleccionados en las bases de datos full text: PubMed, SciELO y Scholar Google, mediante la búsqueda por: "entrenamiento concurrente, ejercicio concurrente, plasticidad muscular, ejercicio físico" en portugués, inglés y español.

Como criterios de inclusión se consideraron todas las formas de vincular conocimientos sobre el contenido seleccionado, que contenía los descriptores deseados, publicados entre 2005 a 2019, con disponibilidad de material sin costo, y que tenía contenido científico consistente; y como criterio de exclusión, fuentes que no permitieron la aplicación completa del método elegido o quién no investigó el tema abordado en el mismo alcance de este estudio.

Del total de referencias obtenidas, y después de la depuración de los criterios de inclusión y exclusión, se utilizaron 19 referencias en el presente estudio por libre elección del autor.

\section{Desarrollo}

Estímulos para mejorar la flexibilidad, la resistencia muscular localizada, el condicionamiento cardiorrespiratorio y también la fuerza son importantes, pues tales valencias figuran como componentes de la aptitud física relacionada a la salud (ACSM, 2017), y están inversamente relacionadas con el riesgo de mortalidad (RosaGuillamón, 2018). De esta forma, se recomienda un programa de entre- namiento integral que contemple los distintos aspectos mencionados (Garber et al., 2011).

La inclusión del entrenamiento de fuerza combinado con el ejercicio cardiorrespiratorio en un mismo programa de entrenamiento se denomina entrenamiento concurrente (EC) (De Souza et al., 2013; Della Corte et al., 2019; Jesus et al., 2016; Wilson et al., 2012). Esta estrategia de combinar diferentes modalidades se realiza ya que es posible adquirir simultáneamente los beneficios de cada una de ellas (de Sena Martins et al., 2017 ; Rosa, Fortes, \& Mello, 2016).

Sin embargo, se cree que las adaptaciones ocasionadas por el entrenamiento de la fuerza y por el ejercicio cardiorrespiratorio pueden ser consideradas como antagónicas, reduciendo el rendimiento de esas capacidades en un programa donde son demandadas simultáneamente (Blagrove, 2013; De Souza, et al., 2013; Paulo, 2005). Este efecto es denominado interferencia negativa (Küüsmaa-Schildt et al., 2017).

Las adaptaciones inducidas por el ejercicio en las fibras del músculo esquelético son específicas para cada tipo de estímulo proporcionado por sus distintas modalidades. Estas adaptaciones son mediadas por distintas vías de señalización (Powers \& Howley, 2014).

El entrenamiento de fuerza provoca una perturbación mecánica en la 
célula muscular, lo que provoca la activación de vías de señalización que conducen a una respuesta de síntesis proteica e hipertrofia (Blagrove, 2013). Cuando los músculos se contraen, la fuerza mecánica impuesta sobre la fibra muscular puede desencadenar procesos de señalización para la promoción de la adaptación. El estiramiento pasivo de una fibra muscular, por ejemplo, estimula diversas vías bioquímicas señalizadoras, como la activación de proteínas quinasas y las cascadas de señalización de factor de crecimiento de insulina (Powers \& Howley, 2014).

Las sesiones de ejercicios prolongados y con gran cantidad de contracciones musculares, como el ejercicio cardiorrespiratorio, activan indicadores metabólicos relacionados a la depleción energética, captación y liberación de iones calcio del retículo sarcoplasmático, y estrés oxidativo en las células. La quinasa activada con monofosfato de adenosina (AMPK) es un potente mensajero con función de monitor de la homeostasis energética. Cuando se activa, AMPK modula la liberación del peroxisome proliferator co-activator-7 $(P C C-7 \alpha)$, que se relaciona con calcio-calmodulinadependientes quinasas (Ca2 + CaM) aumentando la función mitocondrial para mejorar la capacidad aerobia (Blagrove, 2013; Powers \& Howley, 2014).
Sin embargo, esta vía de señalización estimulada por el ejercicio cardiorrespiratorio es capaz de causar interferencia en la vía estimulada por el entrenamiento de fuerza. El AMPK puede inhibir la acción entre Pi-3K y mTOR a través del complejo de esclerosis tuberosa (TSC) suprimiendo la síntesis proteica inducida por el entrenamiento de fuerza (Brook et al. 2016; Mounier, Théret, Lantier, Foretz, \& Viollet, 2015; Powers \& Howley, 2014), como muestra la Figura 1 adaptada de Blagrove, R. (2013). 


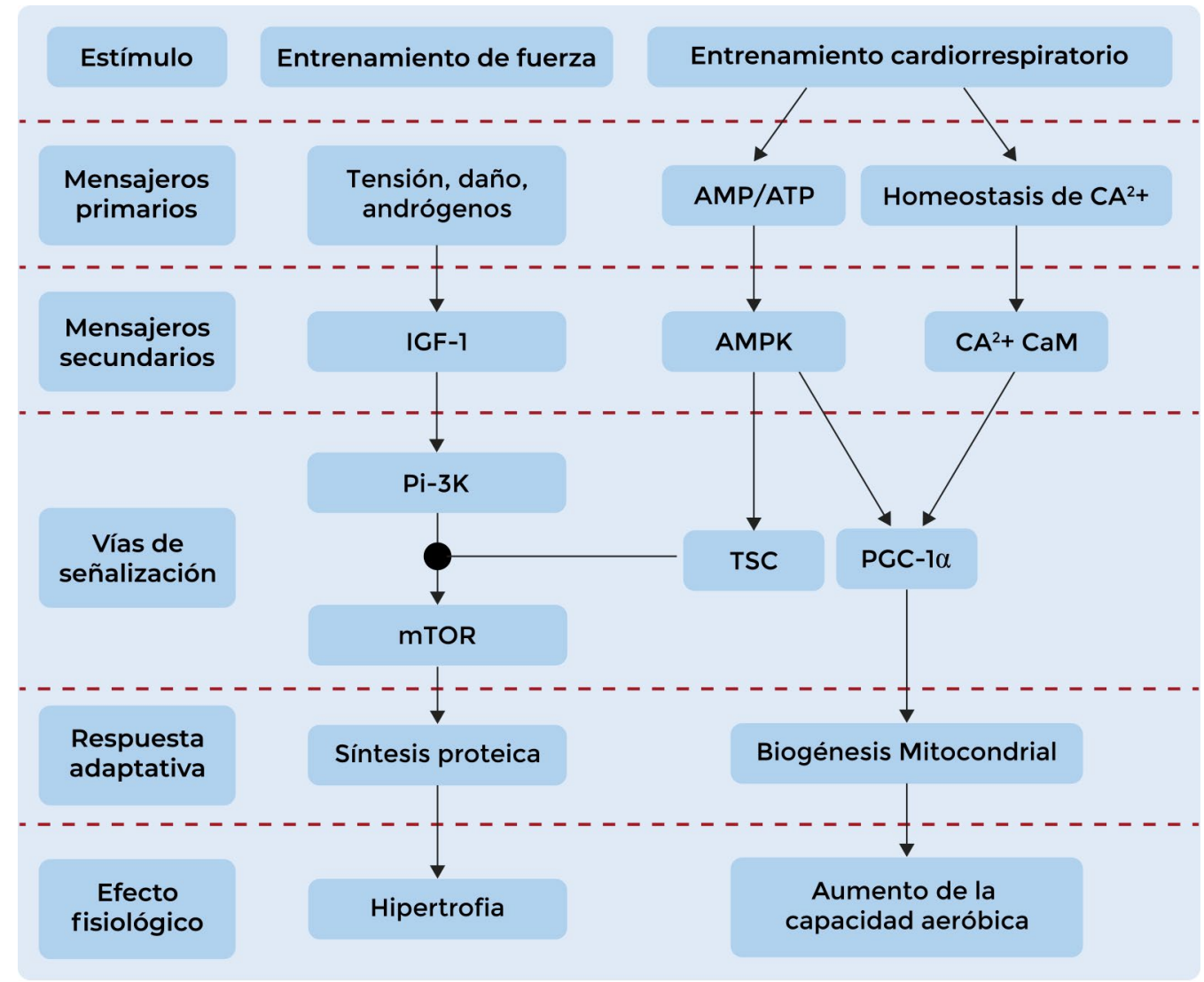

Leyenda: IGF-7 = factor de crecimiento de insulina-7; PI-3K = quinasa dependiente de fosfoinositido-3; $\mathrm{mTOR}=$ mammalian target of rapamycin; $\mathrm{Ca} 2+\mathrm{CaM}=$ quinasas dependientes de calciocalmodulina; $\mathrm{AMPK}=$ quinasa activada con monofosfato de adenosina; $\mathrm{PGC}-1 \alpha=$ proliferador de peroxisoma coactivador- $\alpha$; TSC2 = complejo de esclerosis tuberosa.

Figura 7. Vías de señalización molecular activadas en respuesta al entrenamiento de fuerza y entrenamiento cardiorrespiratorio.

El círculo negro indica una fuente probable de interferencia de la interacción entre las vías de señalización. Esta interacción explicaría la interferencia negativa observada en los programas de entrenamiento concurrente.

De esta forma, en el momento de planificación de un programa de entrenamiento de fuerza como el ejercicio cardiorrespiratorio serán prescritos, algunos aspectos deben ser observados en el intento de minimizar la interferencia negativa.

Primero, es necesario observar el objetivo primario del programa de entrenamiento o de determinado ciclo de la preparación. Aparentemente, la interferencia negativa del EC no se centra en las adaptaciones relacionadas al ejercicio cardiorrespiratorio. En este sentido, cuando el objetivo primario es el perfeccionamiento del 
$\mathrm{VO}_{2}$ máx, tanto el ejercicio cardiorrespiratorio realizado de forma aislada como el EC pueden ser realizados sin interferencia sobre las posibles ganancias en los niveles de la variable.

En contraste, en relación a la interferencia sobre las adaptaciones asociadas al entrenamiento de fuerza, aparentemente, cuanto mayor sea la duración de las sesiones de ejercicio cardiorrespiratorio, y cuanto mayor sea la frecuencia semanal de tales sesiones, mayor será el impacto sobre las ganancias de fuerza e hipertrofia. En el sentido de minimizar tales efectos, las sesiones de ejercicio cardiorrespiratorio deben realizarse por un máximo de tres veces por semana con una duración máxima de 30 minutos (Wilson, et al., 2012). Además, los datos sugieren que la interferencia negativa puede ser minimizada cuando las sesiones de entrenamiento de fuerza y ejercicio cardiorrespiratorio se separan por más de ocho horas de intervalo (Küüsmaa-Schildt, et al., 2017).

\section{Conclusión}

El músculo esquelético presenta la capacidad de adaptarse a los estímulos generados por las distintas modalidades del ejercicio físico. Esta capacidad es denominada plasticidad, donde estímulos distintos generarán adaptaciones específicas.

El entrenamiento concurrente combina los estímulos del entrenamiento de fuerza y del ejercicio car- diorrespiratorio en un mismo programa de entrenamiento. Pero, a pesar de la posibilidad de obtención de beneficios de las distintas modalidades, hay también posibilidad de minimización de las ganancias estimuladas por cada una de ellas, pues las vías de señalización intracelulares son específicas y antagónicas. Además, la vía de señalización estimulada por el ejercicio cardiorrespiratorio es capaz de inhibir la vía responsable por la síntesis proteica disminuyendo sus efectos. Este fenómeno es conocido como interferencia negativa.

De esta manera, en el intento de minimizar esta interferencia negativa en las adaptaciones de una modalidad sobre la otra, principalmente cuando el objetivo primario del programa de entrenamiento es la obtención de ganancias significativas en la fuerza o la hipertrofia, debe haber mayor control sobre la frecuencia semanal y la duración de cada sesión del entrenamiento cardiorrespiratorio.

Para aumentar el cuerpo de conocimiento científico sobre este tema, el autor sugiere que las investigaciones futuras aborden otros puntos que pueden arrojar más luz sobre el fenómeno de la interferencia negativa del entrenamiento concurrente, como las posibles formas de manipulación de las variables de prescripción del entrenamiento y también la influencia de las vías metabólicas de suministro de energía para la contracción muscular durante el ejercicio. 


\section{REFERENCIAS BIBLIOGRÁFICAS}

ACSM. (2017). Guidelines For Exercise Testing and Prescription. 10th ed. Philadelphia: Lippincott WiIliams \& Wilkins.

Blagrove, R. (2013). Programmes of concurrent strength and endurance training: how to minimise the interference effect. Part 1: Evidence and mechanisms of interference. Professional Strength and Conditioning, 37, 7-74. Recuperado de https:// www.researchgate.net/publication/319464447_Programmes_of_concurrent_strength_ and_endurance_training_how_ to_minimise_the_interference_ effect_Part_1_Evidence_and_ mechanisms_of_interference

Brook, M., Wilkinson, D., Phillips, B., Perez-Schindler, J., Philp, A., Smith, K., \& Atherton, P. (2016). Skeletal muscle homeostasis and plasticity in youth and ageing: impact of nutrition and exercise. Acta Physiologica, 216(1), 15-41. doi: 10.1711/apha.12532

de Sena Martins, A., Duarte, D. N. D. B. B., de Paula Silva, G., de Oliveira Leal, S. M., Alías, A., Rodrigues, L. C., \& Rosa, G. (2017). Força muscular em mulheres praticantes de treinamento de força e de treinamento concorrente: um estudo descritivo comparativo. Revista de Educação Física/ Journal of Physical Education,
86(2), 88-94. Recuperado de http://189.38.70.15/index.php/revista/article/view/221

De Souza, E., Tricoli, V., Roschel, H., Brum, P. C., Bacurau, A., Ferreira, J. C. B. et al. (2013). Molecular adaptations to concurrent training. International Journal of Sports Medicine, 34(03), 207213. DOI: 10.1055/s-0032-1312627

Della Corte, J., Rangel, L., de Souza Vale, R. G., de Mello, D. B., Marcos-Pardo, P. J., Rosa, G. (2019). ¿Afecta el entrenamiento intervalado de alta intensidad (HIIT) al desempeño en el entrenamiento de la fuerza? Archivos de Medicina del Deporte $N^{\circ}$ 189, 32(1), 8-12. Recuperado de http://archivosdemedicinadeldeporte.com/articulos/upload/ FEMEDE_189.pdf

Farías, M.C., Borba-Pinheiro, C., Oliveira, M., \& Vale, R.G.S. (2014). Efectos de un programa de entrenamiento concurrente sobre la fuerza muscular, flexibilidad y autonomía funcional de mujeres mayores. Ciencias De La Actividad Física UCM, 15(2), 13-24. Recuperado de http://revistacaf. ucm.cl/article/view/50 
Garber, C,E., Blissmer, B., Deschenes, M.R., Franklin, B.A., Lamonte ,M.J., Lee, I.M., Nieman, D.C., Swain, D.P., American College of Sports Medicine. (2011). American College of Sports Medicine position stand. Quantity and quality of exercise for developing and maintaining cardiorespiratory, musculoskeletal, and neuromotor fitness in apparently healthy adults: guidance for prescribing exercise. Medicine \& Science Sports \& Exercise, 43(7), 1334-59. doi: 10.1249/MSS.0b013e318213fefb.

Hoppeler, H. (2016). Molecular networks in skeletal muscle plasticity. Journal of Experimental Biology, 219(2), 205-213. doi:10.1242/jeb.128207

Jesus, M. A., Mello, D. B., Alias, A., Ribeiro, J., Nunes, K., \& Rosa, G. (2016). Efeito agudo do exercício cardiorrespiratório sobre o desempenho da força em membros inferiores. Revista de Educação Física, 85(4). Recuperado de http://189.38.70.15/index.php/revista/article/view/197/pdf_64

Küüsmaa-Schildt, M., Eklund, D., Avela, J., Rytkönen, T., Newton, R., Izquierdo, M., \&Häkkinen, K. (2017). Neuromuscular adaptations to combined strength and endurance training: order and timeof-day. International Journal of Sports Medicine, 38(09), 707716. DOI:10.1055/s-0043-101376
Monteiro-Lago, T., Cardoso, MD., Henriques, I., Mello, D.B., Fortes, M.S.R., Vale, R.G.S., \& Rosa, G. (2019). Impact of eight weeks of concurrent training on obesityrelated biochemical parameters and cardiometabolic risk factors: a case report. Advances in Obesity, Weight Management \& Control, 9(4), 98-103. DOl: 10.15406/aowmc.2019.09.00281

Mounier, R., Théret, M., Lantier, L., Foretz, M., \& Viollet, B. (2015). Expanding roles for AMPK in skeletal muscle plasticity. Trends in Endocrinology \& Metabolism, 26(6), 275-286. doi: 10.1016/j. tem.2015.02.009

Paulo, A. (2005). Efeito do treinamento concorrente no desenvolvimento da força motora e da resistência aeróbia. Revista Mackenzie de Educação Física e Esporte, 4(4), 145-154. Recuperado de http://www.bbheart. com.br/Trein_Conc.pdf

Powers, S., \& Howley, E. (2014). Fisiologia do Exercício: teoria e aplicação ao condicionamento e ao desempenho. 8 ed. Barueri: Manole.

Rosa, G., Dantas, E., Biehl, C., e Silva, H. D. C., Montano, M. A. E., \& De Mello, D. B. (2012). Leptin, cortisol and distinct concurrent training sequences. International journal of sports medicine, 33(03), 177-180. DOI: 10.1055/s0031-1298002 
Rosa, G., Fortes, M. S. R., Mello, D. B. Wilson, J., Marin, P., Rhea, M., Wilson, (2016). Concurrent training deS., Loenneke, J., \& Anderson, J. creases cortisol but not zinc (2012). Concurrent training: A concentrations: effects of dismeta-analysis examining intinct exercise protocols. Scientifica. Recuperado de https://doi. org/10.1155/2016/7643016 terference of aerobic and resistance exercise. J Strength Cond Res, 26(8), 2293-2307. doi: 10.1519/JSC.0b013e31823a3e2d

Rosa-Guillamón, A. (2018). Análisis de la relación entre salud, ejercicio físico y condición física em escolares y adolescentes. Revista Ciencias de la Actividad Física UCM, 20(1), 1-15. Recuperado de https://doi.org/10.29035/ rcaf.20.1.1

\section{Dirección para correspondencia}

Guilherme Rosa

Pós Doutorado em Fisiologia do Exercício E desempenho Humano - EsEFEx/RJ.

Grupo de Pesquisas em Exercício Físico e Promoção da Saúde - UCB/RJ;

Faculdade Bezerra de Araújo - FABA/RJ.

https://orcid.org/0000-0001-9172-6461

Avenida dos Mananciais, 1501, Bloco 11, Ap

401. CEP: 22720-400.

Rio de Janeiro - RJ. Brasil.

Contacto:

grfitness@hotmail.com

Recibido: 26-08-2019

Aceptado: 08-11-2019 